\title{
Direct observation of the vortex core magnetization and its dynamics
}

\author{
K. W. Chou, ${ }^{\text {a) }}$ A. Puzic, H. Stoll, D. Dolgos, and G. Schütz \\ Max-Planck-Institut für Metallforschung, 70569 Stuttgart, Germany \\ B. Van Waeyenberge and A. Vansteenkiste \\ Department of Subatomic and Radiation Physics, Ghent University, 9000 Gent, Belgium
}

T. Tyliszczak

Advanced Light Source, LBNL, Berkeley, California 94720

\author{
G. Woltersdorf and C. H. Back \\ Institut für Experimentelle und Angewandte Physik, Universität Regensburg, 93040 Regensburg, Germany
}

(Received 27 February 2007; accepted 18 April 2007; published online 18 May 2007)

\begin{abstract}
Square-shaped thin film structures with a single magnetic vortex were investigated using a scanning transmission $\mathrm{x}$-ray microscope. The authors report on the direct observation of the vortex core in $500 \times 500 \mathrm{~nm}^{2}, 40 \mathrm{~nm}$ thick soft magnetic Ni-Fe samples. The static configuration of the vortex core was imaged as well as the gyrotropic motion of the core under excitation with an in-plane alternating magnetic field. This enabled them to directly visualize the direction of the out-of-plane magnetization in the vortex core (up or down). The reversal of the core was effected by short bursts of an alternating magnetic field. An asymmetry appears in the core's trajectory for its orientation pointing up and down, respectively. (C) 2007 American Institute of Physics. [DOI: $10.1063 / 1.2738186]$
\end{abstract}

The magnetic properties of patterned ferromagnetic thin film structures are recently attracting considerable attention. The arrangement of magnetic moments in micro- and nanostructures and their excitations are key subjects to be investigated. Micromagnetic calculations were employed to predict the magnetic equilibrium state of such systems, and have been verified experimentally. The dynamics of the magnetization in these small elements, on the other hand, is much more challenging. Such investigations are not only interesting for modern magnetism theory but are also important for developing high density magnetic recording media where fast switching speeds are necessary.

Micron- or submicron-sized magnetic patterns minimize their stray field energy by forming regions of inhomogeneous magnetization, e.g., domain walls. In thin film ferromagnetic structures, the competing contributions from the exchange energy between neighboring spins and long-range dipoledipole interactions can result in a very stable magnetic vortex configuration, ${ }^{1}$ also called Landau structure in squares. The stability of such structures has already been investigated and is well understood. ${ }^{4-8}$ The uniformly magnetized domains in a Landau pattern are separated by $90^{\circ}$ Néel walls and form an in-plane flux closure [yellow arrows in Fig. 1, panel (a)]. The curling magnetization at the center of the element turns out of the plane avoiding a singularity and forming in this region the vortex core [red arrow in Fig. 1, panel (a)], which plays a key role in the magnetization dynamics. ${ }^{2,3}$ For the experimental study of magnetic vortex structures magnetic force microscopy, ${ }^{9}$ Lorentz microscopy, ${ }^{9}$ spin-polarized scanning tunneling microscopy, ${ }^{10}$ magnetic $\mathrm{x}$-ray microscopy, ${ }^{11}$ and magneto-optical techniques ${ }^{5,6,12,13}$ can be deployed. Study of the details in the dynamic response of a vortex structure to externally applied magnetic field pulses and continuous excitations was only possible with the advent of time-resolved magnetic transmission x-ray

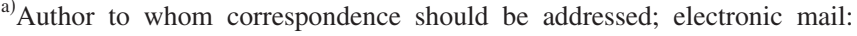
chou@mf.mpg.de microscopy ${ }^{14,15,17}$ and photoemission electron microscopy. ${ }^{16}$

In the current work we report on the direct observation of a magnetic vortex core and its dynamic behavior under influence of an in-plane alternating magnetic field. Squareshaped $500 \times 500 \mathrm{~nm}^{2}, 40 \mathrm{~nm}$ thick Permalloy $\left(\mathrm{Ni}_{80} \mathrm{Fe}_{20}\right)$ samples (A and B) were investigated. The magnetic microstructures were patterned by e-beam lithography onto a $10 \mu \mathrm{m}$ wide and $150 \mathrm{~nm}$ thick $\mathrm{Cu}$ stripline, and the samples and the stripline structure were deposited on a $100 \mathrm{~nm}$ thin $\mathrm{Si}_{3} \mathrm{~N}_{4}$ membrane. The complete structure was capped with a $2 \mathrm{~nm} \mathrm{Al}$ protective coating. The alternating current $I_{\text {sin }}$ in the stripline induces a magnetic field $H_{\sin }=H_{0} \sin (2 \pi f t)$ in the plane of the sample perpendicular to the current direction. The time dependent spatial distribution of the magnetization $\mathbf{M}(\mathbf{r}, t)$ was imaged by a stroboscopic measurement technique $^{14}$ using the scanning transmission $\mathrm{X}$-ray microscope (STXM) at the Advanced Light Source (ALS, BL 11.0.2). ${ }^{18}$ The STXM beamline, equipped with an elliptical undulator and a high resolution monochromator, allows an easy and accurate adjustment of the energy and the polariza-
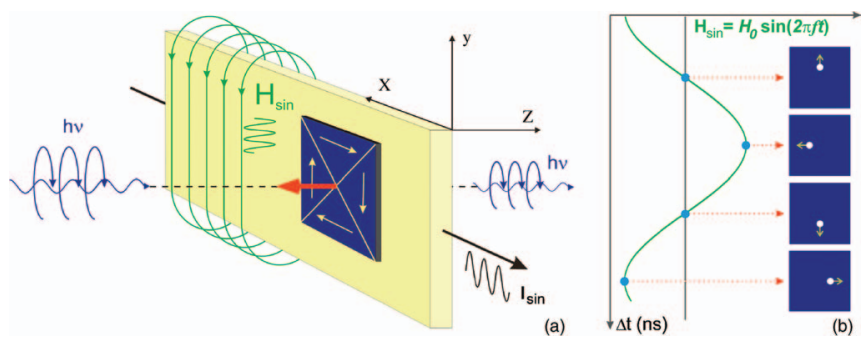

FIG. 1. (Color) Illustration of the sample setup and the stroboscopic measuring technique used for the time-resolved experiments. Panel (a) shows the vortex structure on a stripline. The yellow arrows illustrate the in-plane distribution of the magnetization forming a closed flux around the out-ofplane vortex core, depicted with a red arrow. The sample is placed perpendicularly with respect to the incoming x-ray flashes. An ac $I_{\sin }$ is sent through the stripline, generating an alternating magnetic field $H_{\sin }$. This in-plane field induces a gyrotropic motion in the vortex structure which can be imaged by probing at specific phases of the applied field [panel (b)]. 


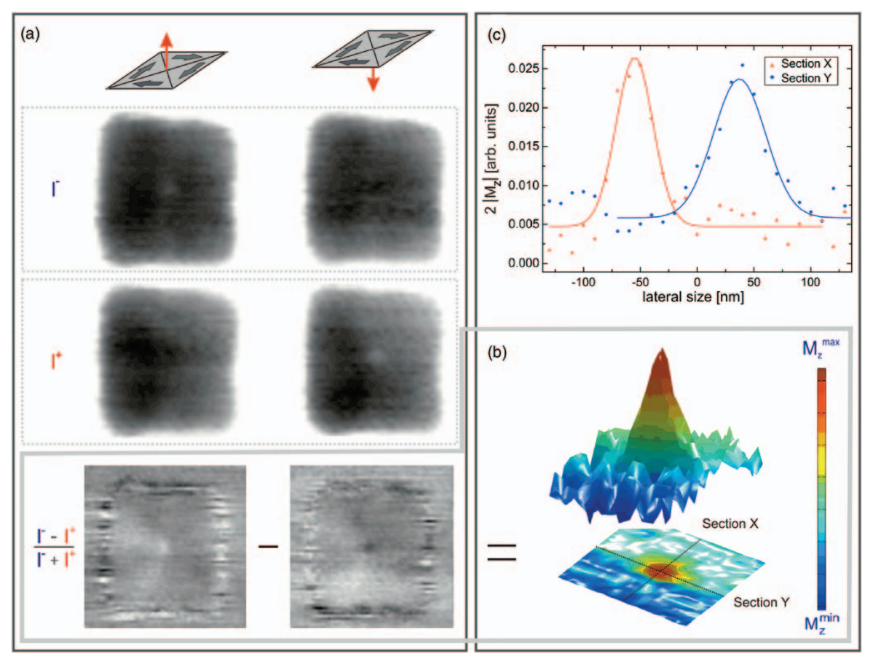

FIG. 2. (Color) Panel (a) shows the static configuration of a vortex structure (sample A: $500 \times 500 \mathrm{~nm}^{2}, 40 \mathrm{~nm}$ thick) with a vortex core pointing up (left column) and down (right column). In the first row, images were taken for negative polarization of the photons $\left(I^{-}\right)$while images with opposite polarization $\left(I^{+}\right)$are shown in the second row. The "dichroic image" is depicted in the third row. A white or black spot can be observed, corresponding to a vortex core pointing up or down, respectively. The two dichroic images were subtracted from one another and a $3 \mathrm{D}$ image is given in panel (b) $\left(200 \times 200 \mathrm{~nm}^{2}\right.$ cut $)$. The distributions along sections $X$ and $Y$ are plotted in panel (c) with the corresponding Gaussian fits in order to estimate the size of the vortex core.

tion of the x-rays. The contrast mechanism used for the imaging of the magnetic structures is the $\mathrm{x}$-ray magnetic circular dichroism (XMCD). ${ }^{19}$ The absorption of circularly polarized photons is proportional to the projection of the magnetization on the photon propagation direction; thus for the observation of the out-of-plane magnetization component, the sample is placed perpendicular with respect to the incoming x-rays. The x-rays are focused by a Fresnel zone plate to a spot of about $30 \mathrm{~nm}$ on the magnetic sample. The transmitted x-ray intensity is detected by a photodetector (avalanche photodiode). The sample is scanned with a high resolution scanning stage under interferometric control. Images were acquired at the $L_{3}$ absorption edge of $\mathrm{Ni}$ $(852.7 \mathrm{eV})$, where XMCD gives a high magnetic contrast. The response of the magnetic structure was monitored at different phases of the excitation signal between consecutive images. Panel (a) of Fig. 1 shows a sketch of the sample setup, and the concept of resonant sine excitation synchronized with the probing $\mathrm{x}$-ray flashes is sketched in panel (b). A time resolution of less than $100 \mathrm{ps}$ is given by the inherent time structure of the synchrotron radiation. More details can be found in Ref. 15.

To study the static configuration, an image $\left(I^{-}\right)$was taken from sample A. Subsequently a second image $\left(I^{+}\right)$was taken by reversing the polarization of the x-rays, and a "dichroic image" was deduced from both images using the relation $\left(I^{-}-I^{+}\right) /\left(I^{-}+I^{+}\right)$. The adopted method is hereby only sensitive to the out-of-plane component of the magnetization. A dark or bright spot can clearly be observed in the two images recorded for a vortex core pointing up and down, respectively, in panel (a) of Fig. 2. A zone plate allowing a lateral resolution of $30 \mathrm{~nm}$ was used to take the images. Nevertheless, the large magnetic contrast of the XMCD effect provides a magnetic signal sufficient to observe the vortex core directly. core directly.
Downloaded 12 Sep 2007 to 132.199.211.8. Redistribution subject to AlP license or

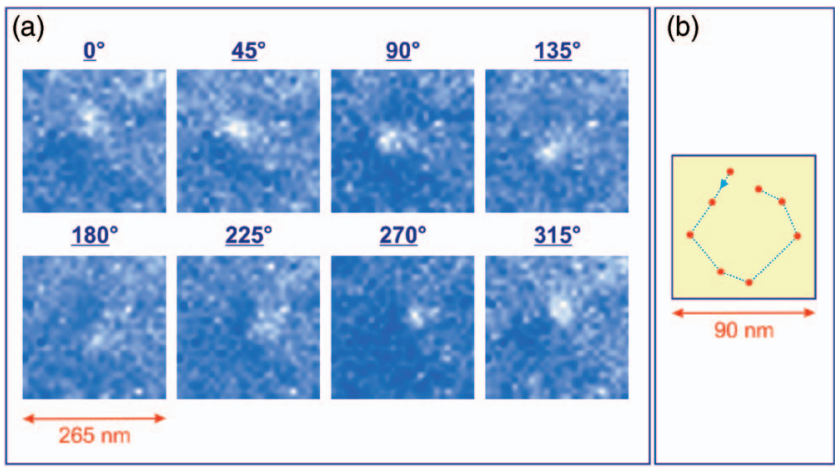

FIG. 3. (Color) Dynamic response of a vortex structure (sample B: $500 \times 500 \mathrm{~nm}^{2}, 40 \mathrm{~nm}$ thick) by applying an in-plane alternating magnetic field $\left(f=437.5 \mathrm{MHz}, H_{0}=590 \mathrm{~A} / \mathrm{m}\right)$. Panel (a) shows the out-of-plane magnetic contrast of the Landau structure at different phases with respect to the external field. The images are cuts $\left(265 \times 265 \mathrm{~nm}^{2}\right)$ from the complete structure. The position at the different phases is depicted in panel (b) $\left(90 \times 90 \mathrm{~nm}^{2}\right)$.

The reversal of the direction of the magnetization in the vortex core was carried out with a short burst of an alternating magnetic field ${ }^{17}$ and is observed as a change from a white to a black dot in the middle of the structure. The two dichroic images, corresponding to a vortex core pointing up and down, respectively, were thereafter subtracted from one another. A three-dimensional (3D) image of this "differential representation" of the vortex core is shown in panel (b). The size of the vortex core can be estimated by fitting a Gaussian distribution through the orthogonal sections $X$ and $Y$. Full widths at half maximum are found to be approximately 38 and $54 \mathrm{~nm}$ in the $X$ and $Y$ sections, respectively [see panel (c)]. The additional broadening along the $Y$ axis originates in an astigmatism due to a small misalignment in the optics. Considering the instrumental resolution, a vortex core width of $22.5 \mathrm{~nm}$ can be estimated. This is close to the width of $21 \mathrm{~nm}$ obtained from micromagnetic simulations. ${ }^{20}$ In the same way, the maximum magnetic contrast can be estimated for the out-of-plane signal of the core from inset (c). A value of $26 \%$ is found which corresponds well with the dichroic signal of Ni in Permalloy [about 25\% (Refs. 21 and 22)].

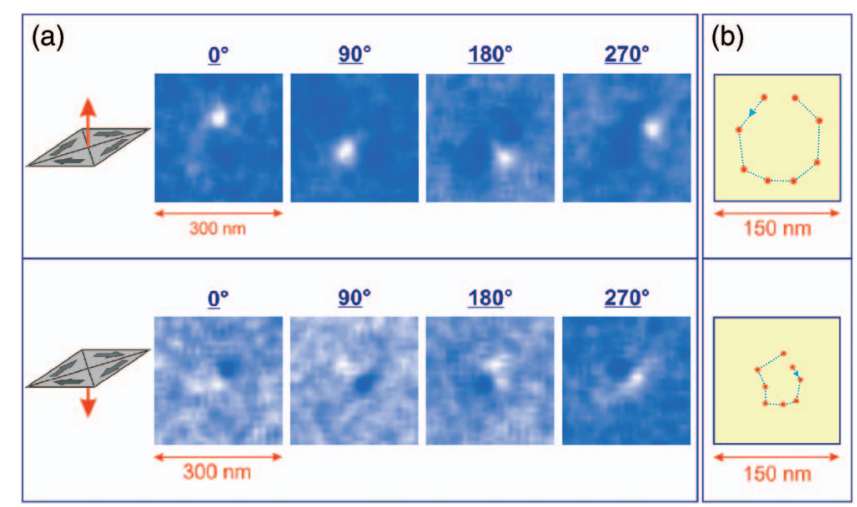

FIG. 4. (Color) Dynamic response of a vortex structure (sample A: $500 \times 500 \mathrm{~nm}^{2}, 40 \mathrm{~nm}$ thick) due to an in-plane alternating magnetic field $\left(f=437.5 \mathrm{MHz}, H_{0}=710 \mathrm{~A} / \mathrm{m}\right.$ ) with a vortex core pointing up (upper row) and down (lower row). Panel (a) shows the out-of-plane magnetic contrast of the Landau structure at different phases of the external field (uneven phases are not shown). The images are cuts $\left(300 \times 300 \mathrm{~nm}^{2}\right)$ from the complete structure. The position at the different phases is depicted in panel (b) 
The dynamic response was studied by applying an ac through the stripline, inducing an in-plane alternating magnetic field, with an amplitude of $590 \mathrm{~A} / \mathrm{m}$. The frequency $f$ was set to $437.5 \mathrm{MHz}$, which is close to the resonance frequency. The vortex core will thus perform a gyrotropic motion around the center of the element. Two sequences of images $\left(I^{-}\right.$and $\left.I^{+}\right)$were recorded for sample B with opposite polarization of the X-rays. A sequence of dichroic images was again deduced from both sequences using the relation $\left(I^{-}-I^{+}\right) /\left(I^{-}+I^{+}\right)$and is shown in panel (a) of Fig. 3. A white spot can be distinguished representing the out-of-plane magnetization contrast at different phases of the applied in-plane magnetic field. The contrast fades at certain phases (most noticeable at $180^{\circ}$ and $225^{\circ}$ ), which can be explained by a deformation of the vortex core due to the movement as noticed in micromagnetic calculations. ${ }^{17,23}$ This deformation with opposite magnetization with respect to the core, cannot be resolved due to the limited resolution but will reduce the observed magnetic contrast. The position of the core at the different phases was extracted and is shown in panel (b), showing a counterclockwise motion of the vortex structure. An average velocity of the vortex core of about $93 \mathrm{~m} / \mathrm{s}$ can be deduced by fitting an ellipse through the trajectory.

The vortex motion was also recorded for sample A, with a magnetic field amplitude of $710 \mathrm{~A} / \mathrm{m}$ and a frequency of 437.5 MHz. Two sequences of images were recorded at a fixed polarization of the x-rays but for the opposite direction of the magnetization in the core, respectively. In order to observe the vortex core gyration, the data were divided by an average of all images to improve the signal-to-noise ratio. The two sequences reveal a different sense of the vortex core gyration [Fig. 4, panel (a)]. The reversal of the vortex core magnetization was carried out with a short burst of the alternating magnetic field. ${ }^{17}$ The position of the vortex core at the different phase angles with respect to the alternating magnetic field is shown in panel (b). The vortex core moves counterclockwise for the vortex core pointing up (bright contrast) and clockwise when pointing down (dark contrast). One also notices the large difference in extend of the gyrotropic vortex trajectory for the two cases. Beside the change in the sense of rotation of the vortex core, a distinct phase difference occurs which can be seen based on symmetry considerations. The phase of the trajectory for a core pointing down should be coinciding with the case of a core pointing up after mirroring along the vertical axis. A phase shift can now be observed comparing the corresponding core positions for both trajectories. As the excitation frequency remains the same for both core polarizations, this indicates a slightly different resonance frequency for the gyrotropic motion, respectively. Both these observations indicate that the vortex structure is moving in a different potential for the two opposite core polarizations. This is not expected in perfectly symmetric samples with smooth surfaces. The occurrence of the asymmetry might be attributed to local imperfections and rough surfaces in the thin film, influencing differently the motion for the vortex core pointing up and down, respectively. The velocity of the vortex core was also deduced from the trajectories. For the vortex core pointing up, a speed of $140 \mathrm{~m} / \mathrm{s}$ was calculated, while a much smaller speed of $82 \mathrm{~m} / \mathrm{s}$ was found when the vortex core is pointing down.

In this work, the vortex core in ferromagnetic Permalloy Landau patterns was imaged directly by means of a STXM.
The static vortex core was visualized and its size and magnetic contrast were estimated. The vortex core gyration, excited by an in-plane alternating magnetic field, was imaged as well, confirming directly that the out-of-plane magnetization in the vortex core is reversed when the gyration sense of the vortex structure changes its direction. A strong asymmetry of the vortex core trajectories and the phase were found for the vortex core pointing up and down, respectively.

The authors would like to acknowledge the helpful discussions with Heinz Dieter Carstanjen, Manfred Fähnle, and Kai Fauth; and Dieter Weiss for the use of the clean room facilities. Financial support by the DFG through the priority program "Ultrafast Magnetisation Processes" is gratefully acknowledged. The Advanced Light Source is supported by the Director, Office of Science, Office of Basic Energy Sciences, of the U.S. Department of Energy.

${ }^{1}$ A. Hubert and R. Schäfer, Magnetic Domains: The Analysis of Magnetic Microstructures (Springer, Berlin, 1998).

${ }^{2}$ D. L. Huber, Phys. Rev. B 26, 3758 (1982).

${ }^{3}$ K. Guslienko, B. Ivanov, V. Novosad, Y. Otani, H. Shima, and K. Fukamichi, J. Appl. Phys. 91, 8037 (2002).

${ }^{4}$ T. Shinjo, T. Okuno, R. Hassdorf, K. Shigeto, and T. Ono, Science 289, 930 (2000).

${ }^{5}$ R. P. Cowburn, D. K. Koltsov, A. O. Adeyeye, M. E. Welland, and D. M. Tricker, Phys. Rev. Lett. 83, 1042 (1999).

${ }^{6}$ V. Novosad, K. Y. Guslienko, H. Shima, Y. Otani, S. G. Kim, K. Fukamichi, N. Kikuchi, O. Kitakami, and Y. Shimada, Phys. Rev. B 65, 060402 (2002)

${ }^{7}$ K. Guslienko, V. Novosad, Y. Otani, H. Shima, and K. Fukamichi, Phys. Rev. B 65, 024414 (2002).

${ }^{8}$ J. Shibata and Y. Otani, Phys. Rev. B 70, 012404 (2004).

${ }^{9}$ J. Raabe, R. Pulwey, R. Sattler, T. Schweinbock, J. Zweck, and D. Weiss, J. Appl. Phys. 88, 4437 (2000).

${ }^{10}$ A. Wachowiak, J. Wiebe, M. Bode, O. Pietzsch, M. Morgenstern, and R. Wiesendanger, Science 298, 577 (2002).

${ }^{11}$ J. Stöhr and H. C. Siegmann, Magnetism: From Fundamentals to Nanoscale Dynamics (Springer, New York, 2006).

${ }^{12}$ J. Park, P. Eames, D. Engebretson, J. Berezovsky, and P. Crowell, Phys. Rev. B 67, 020403 (2003).

${ }^{13}$ B. Argyle, E. Terrenzio, and J. Slonczewski, Phys. Rev. Lett. 53, 190 (1984).

${ }^{14}$ H. Stoll, A. Puzic, B. van Waeyenberge, P. Fischer, J. Raabe, M. Buess, T. Haug, R. Höllinger, C. Back, D. Weiss, and G. Denbeaux, Appl. Phys. Lett. 84, 3328 (2004).

${ }^{15}$ A. Puzic, B. Van Waeyenberge, K. W. Chou, P. Fischer, H. Stoll, G. Schütz, T. Tyliszczak, K. Rott, H. Brückl, G. Reiss, I. Neudecker, T. Haug, M. Buess, and C. H. Back, J. Appl. Phys. 97, 10 E704 (2005).

${ }^{16}$ J. Raabe, C. Quitmann, C. H. Back, F. Nolting, S. Johnson, and C. Buehler, Phys. Rev. Lett. 94, 217204 (2005).

${ }^{17}$ B. Van Waeyenberge, A. Puzic, H. Stoll, K. W. Chou, T. Tyliszczak, R. Hertel, M. Fähnle, H. Brückl, K. Rott, G. Reiss, I. Neudecker, D. Weiss, C. H. Back, and G. Schütz, Nature (London) 444, 461 (2006).

${ }^{18}$ A. Kilcoyne, T. Tyliszczak, W. Steele, S. Fakra, P. Hitchcock, K. Franck, E. Anderson, B. Harteneck, E. Rightor, G. Mitchell, A. Hitchcock, L. Yang, T. Warwick, and H. Ade, J. Synchrotron Radiat. 10, 125 (2003).

${ }^{19}$ G. Schütz, W. Wagner, W. Wilhelm, P. Kienle, R. Zeller, R. Frahm, and G. Materlik, Phys. Rev. Lett. 58, 737 (1987).

${ }^{20}$ The object oriented micromagnetic framework (OOMMF) project was used for the micromagnetic simulations. The relaxation was studied for a $500 \times 500 \times 40 \mathrm{~nm}^{3}$ square, subdivided in small cells of the size of $1 \times 1$ $\times 40 \mathrm{~nm}^{3}$. Typical material parameters were used for Permalloy with $M_{s}$ $=8.6 \times 10^{5} \mathrm{~A} / \mathrm{m}$ for the saturation magnetization and $A=1.3 \times 10^{-11} \mathrm{~J} / \mathrm{m}$ for the exchange constant. The magnetocrystalline anisotropy was neglected, and the damping constant was set to 0.5.

${ }^{21}$ C. T. Chen, N. V. Smith, and F. Sette, Phys. Rev. B 43, 6785 (1991).

${ }^{22} \mathrm{D}$. Jiles, Introduction to Magnetism and Magnetic Materials (Chapman and Hall, New York, 1991).

${ }^{23}$ V. Novosad, F. Fradin, P. Roy, K. Buchanan, K. Guslienko, and S. Bader, Phys. Rev. B 72, 024455 (2005). 